\title{
An improved analytical description of inspiralling and coalescing black-hole binaries
}

\author{
Thibault Damour ${ }^{1,2}$ and Alessandro Nagar ${ }^{1,2,3}$ \\ ${ }^{1}$ Institut des Hautes Etudes Scientifiques, 91440 Bures-sur-Yvette, France \\ ${ }^{2}$ ICRANet, 65122 Pescara, Italy \\ ${ }^{3}$ INFN, Sezione di Torino, Via Pietro Giuria 1, Torino, Italy
}

(Dated: October 22, 2018)

\begin{abstract}
We present an analytical formalism, within the Effective-One-Body framework, which predicts gravitational-wave signals from inspiralling and coalescing black-hole binaries that agree, within numerical errors, with the results of the currently most accurate numerical relativity simulations for several different mass ratios. In the equal-mass case, the gravitational wave energy flux predicted by our formalism agrees, within numerical errors, with the most accurate numerical-relativity energy flux. We think that our formalism opens a realistic possibility of constructing a sufficiently accurate, large bank of gravitational wave templates, as needed both for detection and data analysis of (non spinning) coalescing binary black holes.
\end{abstract}

PACS numbers: $04.25 . \mathrm{Nx}, 04.30 .-\mathrm{w}$, 04.30.Db

The opening of gravitational wave (GW) astronomy depends on our theoretical ability at computing, within Einstein's theory of General Relativity, a sufficiently accurate approximation to the GW signal emitted by the premier target of ground-based GW detectors: inspiralling and coalescing binary black holes (BBH's). Indeed, to detect these GW signals, and extract physical information from them, one must correlate the noisy output of the detectors to a very large bank of "GW templates", each template giving an accurate representation of the gravitational waveform emitted by a $\mathrm{BBH}$ with certain physical parameters (notably masses and spins). Recent breakthroughs in Numerical Relativity (NR) 1, 2, 3, 4, [5, 6] have given us access to an accurate knowledge of the waveform emitted during the late inspiral and merger of a sparse sample of $\mathrm{BBH}$ systems (see 7] for a review). However, BBH simulations are time consuming. This precludes the sole use of NR simulations for building the needed bank of GW templates, densely filling the multidimensional space of $\mathrm{BBH}$ physical parameters (masses and spins). It is urgent to have in hands an analytical method able to give a sufficiently accurate representation of the motion of, and the gravitational radiation from, coalescing binary black holes with arbitrary masses and spins. We shall describe here a formalism which hopefully solves this problem, in the case of circularized, non-spinning binary black holes with arbitrary masses $m_{1}, m_{2}$.

The analytical formalism presented here is a significantly improved version of the general Effective-OneBody (EOB) method [8, 9, 10, 11]. The predictions of previous (less accurate) implementations of the EOB method have already been compared, with success, to various types of results from numerical simulations 12 , $13,14,15,16,17,18,19,20,21$. Before explaining the improvements that characterize our formalism, let us recall that the four essential elements of the EOB approach are: (i) a Hamiltonian $H_{\text {real }}$ describing the conservative part of the relative dynamics of the two black holes; (ii) a radiation-reaction force $\mathcal{F}_{\varphi}$ describing the loss of (mechanical) angular momentum, and energy, of the binary system; (iii) the definition of the various multipolar components of the "inspiral-plus-plunge" (metric) waveform $h_{\ell m}^{\text {insplunge }}$; and (iv) the attachment of a subsequent "ringdown waveform" $h_{\ell m}^{\text {ringdown }}$ around a certain (EOB-determined) "merger time" $t_{m}$. The latter fourth facet of the EOB formalism, namely the assumption of a sharp transition, around the BBH merger, between the "plunge" and a ringdown behavior, was inspired by the classic "plunging test-mass" result of [22]. This assumption has been well confirmed by the results of NR simulations 7], and we shall not try here to improve on it. We shall follow here the usual EOB procedure [9] of matching the "insplunge" and "ringdown" waveforms around the EOB merger, and matching, time $t_{m}$, defined as the location of the maximum EOB orbital frequency. [We use the specific procedure of [16, 19], with 5 quasi-normalmodes, and a "comb" of spacing $\delta=2.3 M_{\mathrm{f}}$, where $M_{\mathrm{f}}$ is the mass of the final black hole.]

On the other hand, we propose here to improve the three other basic elements of the EOB formalism in the following way:

(i) The central object entering the relative Hamiltonian, $H_{\text {real }}=M\left[1+2 \nu\left(\hat{H}_{\text {eff }}-1\right)\right]^{1 / 2}$, where $M \equiv m_{1}+m_{2}$, $\mu \equiv m_{1} m_{2} / M, \nu \equiv \mu / M, z_{3}=2 \nu(4-3 \nu)$, and

$$
\hat{H}_{\mathrm{eff}} \equiv \sqrt{p_{r_{*}}^{2}+A(1 / r)\left(1+p_{\varphi}^{2} / r^{2}+z_{3} p_{r_{*}}^{4} / r^{2}\right)}
$$

is the $\mathrm{EOB}$ radial potential $A(u)$ (here $u=1 / r$; the $\mathrm{EOB}$ radial coordinate $r$ is rescaled by $M$, with $G=c=1$; and $p_{r_{*}}$ is canonically conjugated to the EOB "tortoise-like" radial coordinate $r_{*}$, defined in [14]). Current analytical calculations, within the post-Newtonian (PN) formalism, of the dynamics of BBH's have computed the first four terms (3PN approximation) in the (Taylor) expansion of the radial potential $A(u)$ in powers of $u[10,23]$, namely $A^{3 \mathrm{PN}}(u) \equiv 1-2 u+2 \nu u^{3}+\left(94 / 3-41 \pi^{2} / 32\right) \nu u^{4}$. Here we propose to consider the two-parameter class of (ex- 

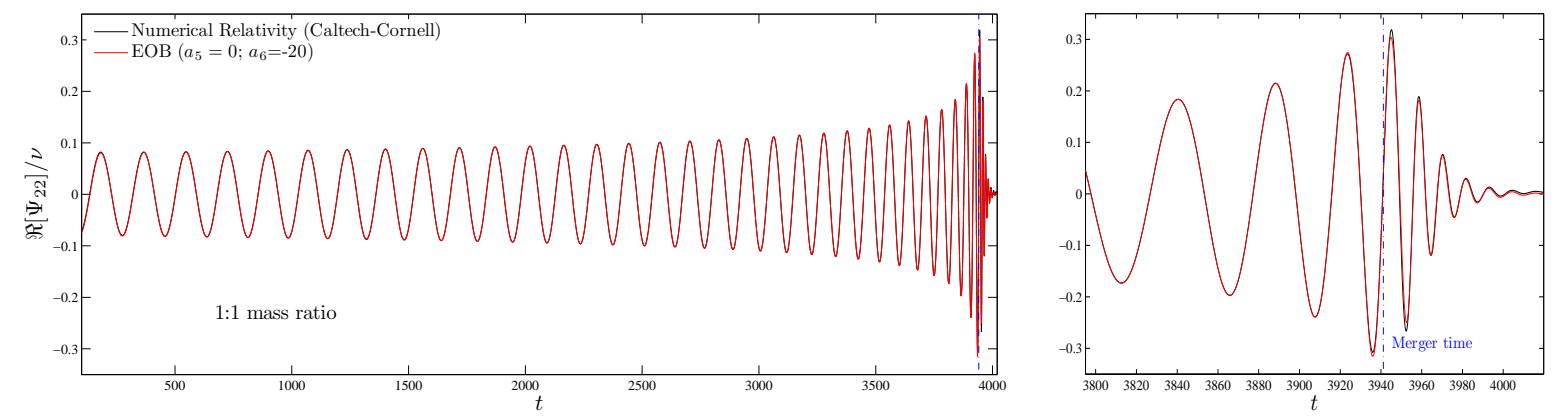

FIG. 1: Equal-mass case: agreement between NR (black online) and EOB-based (red online) $\ell=m=2$ metric waveforms.

tensions and) resummations of $A^{3 \mathrm{PN}}(u)$ defined by

$$
A\left(u ; a_{5}, a_{6}, \nu\right) \equiv P_{5}^{1}\left[A^{3 \mathrm{PN}}(u)+\nu a_{5} u^{5}+\nu a_{6} u^{6}\right],
$$

where $P_{5}^{1}$ denotes a $(1,5)$ Padé approximant. This is a generalization of the one-parameter $\left(a_{5}\right)$ class of $P_{4}^{1}$ Padé-resummed $A$-potentials considered in previous EOB works [11, 17, 18].

(ii) The second (and most novel) improvement that we introduce here concerns the radiation reaction force $\mathcal{F}_{\varphi}$. We make use of the very recent results of 24] concerning an "improved resummation" of post-Newtonian multipolar waveforms. Specifically, we define $\mathcal{F}_{\varphi}$ in the following way ( $\Omega$ denoting the EOB orbital frequency):

$$
\mathcal{F}_{\varphi} \equiv-\frac{1}{8 \pi \Omega} \sum_{\ell=2}^{\ell_{\max }} \sum_{m=1}^{\ell}(m \Omega)^{2}\left|R h_{\ell m}^{(\epsilon)}\right|^{2} .
$$

Here, we shall take $\ell_{\max }=8$ and define the individual multipolar waveforms $h_{\ell m}^{(\epsilon)}$ (where $\epsilon=0,1$ labels the "even" or "odd" parity) in the following way: (a) the leading quadrupolar contribution to $\mathcal{F}_{\varphi}$, i.e. the term $\epsilon=0$ and $\ell=m=2$ in (3), is computed by using the quadrupolar waveform defined in Eq. (4) below; while (b) the subdominant terms (i.e. when, either $\epsilon=1$, or $\epsilon=0$ and $\ell \geq 3), h_{\ell m}^{(\epsilon)}$ are defined by Eq. (1) of [24], together with the other definitions given there (see [25] for details).

(iii) The third improvement introduced here concerns the (dominant) even-parity, quadrupolar $(\epsilon=0$ and $\ell=$ $m=2)$ "insplunge" waveform. We take it in the form

$$
h_{22}=\frac{M \nu}{R} n_{22} x Y^{2,-2}\left(\frac{\pi}{2}, \Phi\right) \hat{H}_{\mathrm{eff}} T_{22} e^{i \delta_{22}} f_{22}(x) f_{22}^{\mathrm{NQC}},
$$

where, for notational simplicity, we have suppressed the parity label $\epsilon=0$. Such a multiplicatively decomposed form of $h_{22}$ was introduced in [16, 18]. See these references, and [20, 24, 25] for the definition of the factors in (4); $f_{22}(x)$ is a modulus correction, here defined (as in [18], where $f_{22}$ was computed, using [26, 27], at the $3^{+2} \mathrm{PN}$ accuracy; Eq. (10) there) as being the following
Padé-resummed function $f_{22}^{\mathrm{Pf}}(x ; \nu)=P_{2}^{3}\left[f_{22}^{\text {Taylor }}(x ; \nu)\right]$. The new ingredient of $h_{22}$ introduced here is the definition of the last factor in Eq. (4), namely an additional "Next-to-Quasi-Circular" (NQC) correction factor of the form [30]

$$
f_{22}^{\mathrm{NQC}}\left(a_{1}, a_{2}\right)=1+a_{1} p_{r_{*}}^{2} /(r \Omega)^{2}+a_{2} \ddot{r} / r \Omega^{2} .
$$

A crucial facet of the new analytical formalism presented here consists in trying to be as predictive as possible by reducing to an absolute minimum the number of "flexibility parameters" entering our theoretical framework. We shall achieve this aim by "analytically" determining the two parameters $a_{1}, a_{2}$ entering (via the NQC factor Eq. (5)) the (asymptotic) quadrupolar EOB waveform $\hat{R} h_{22}^{\mathrm{EOB}}$ (where $\hat{R}=R / M$ ) by imposing: (a) that the modulus $\left|\hat{R} h_{22}^{\mathrm{EOB}}\right|$ reaches, at the EOB-determined "merger time" $t_{m}$, a local maximum, and (b) that the value of this maximum EOB modulus is equal to a certain (dimensionless) function of $\nu, \varphi(\nu)$. We calibrated $\varphi(\nu)$ (independently of the EOB formalism) by extracting from the best current Numerical Relativity simulations the maximum value of the modulus of the Numerical Relativity quadrupolar metric waveform $\left|\hat{R} h_{22}^{\mathrm{NR}}\right|$. Using the data reported in [6] and [20], and considering the "Zerilli-normalized" asymptotic metric waveform $\Psi_{22}=$ $\hat{R} h_{22} / \sqrt{24}$, we found $\varphi(\nu) \simeq 0.3215 \nu(1-0.131(1-4 \nu))$. Our requirements (a) and (b) impose, for any given $A(u)$ potential, two constraints on the two parameters $a_{1}, a_{2}$. We can solve these two constraints (by an iteration procedure) and thereby uniquely determine the values of $a_{1}, a_{2}$ corresponding to any given $A(u)$ potential. In particular, in the case considered here where $A\left(u ; a_{5}, a_{6}, \nu\right)$ is defined by Eq. (2), this uniquely determines $a_{1}, a_{2}$ in function of $a_{5}, a_{6}$ and $\nu$.

Finally, our analytical formalism contains only two analytically undertermined parameters, namely $a_{5}$ and $a_{6}$, which parametrize some flexibility in the Padéresummation of the basic radial potential $A(u)$, connected to the yet uncalculated higher PN contribution [31]. We have first compared the $\left(a_{5}, a_{6}\right)$-dependent predictions made by our formalism to the high-accuracy 


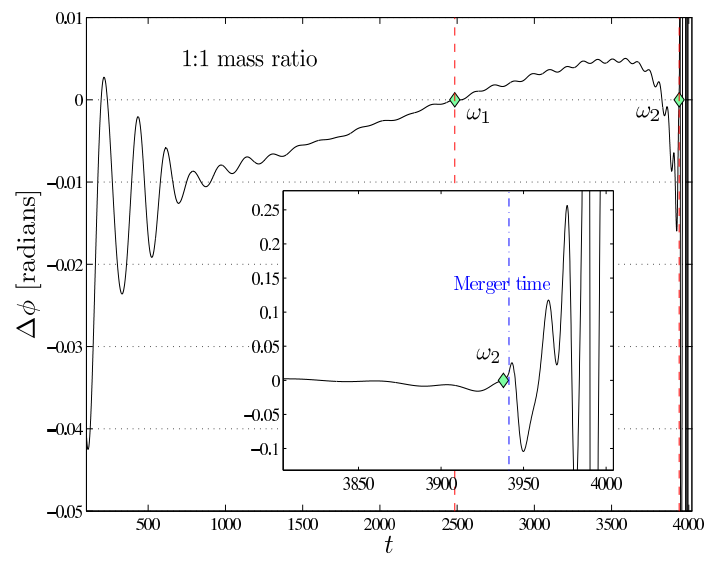

FIG. 2: Phase difference between the analytical and numerical (metric) waveforms of Fig. 1.

waveform from an equal-mass $\mathrm{BBH}(\nu=1 / 4)$ computed by the Caltech-Cornell group [6] (and now made available on the web). We found that there is a strong degeneracy between $a_{5}$ and $a_{6}$ in the sense that there is an excellent EOB-NR agreement for an extended region in the $\left(a_{5}, a_{6}\right)$-plane. More precisely, the phase difference between the EOB (metric) waveform and the CaltechCornell one, considered between GW frequencies $M \omega_{\mathrm{L}}=$ 0.047 and $M \omega_{\mathrm{R}}=0.31$ (i.e., the last $16 \mathrm{GW}$ cycles before merger), stays smaller than 0.02 radians within a long and thin banana-like region in the $\left(a_{5}, a_{6}\right)$-plane. This "good region" approximately extends between the points $\left(a_{5}, a_{6}\right)=(0,-20)$ and $\left(a_{5}, a_{6}\right)=(-36,+520)$. As an example (which actually lies on the boundary of the "good region"), we shall consider here the specific values $a_{5}=0, a_{6}=-20$ (to which correspond, when $\nu=1 / 4$, $\left.a_{1}=-0.036347, a_{2}=1.2468\right)$. We henceforth use $M$ as time unit.

Figure1 1 compares (the real part of) our analytical metric quadrupolar waveform $\Psi_{22}^{\mathrm{EOB}} / \nu$ to the corresponding (Caltech-Cornell) NR metric waveform $\Psi_{22}^{\mathrm{NR}} / \nu$ (obtained by a double time-integration, à la 20], from the original NR curvature waveform $\psi_{4}^{22}$ ). [We used the "twofrequency pinching technique" of [19] with $\omega_{1}=0.047$ and $\omega_{2}=0.31$.] The agreement between the analytical prediction and the NR result is striking, even around the merger (see the close-up on the right). The phasing agreement is excellent over the full time span of the simulation (which covers 32 cycles of inspiral and about 6 cycles of ringdown), while the modulus agreement is excellent over the full span, apart from two cycles after merger where one can notice a difference. A more quantitative assessment of the phase agreement is given in Fig. 2, which plots the $\left(\omega_{1}-\omega_{2}\right.$-pinched $)$ phase difference $\Delta \phi=\phi_{\text {metric }}^{\mathrm{EOB}}-\phi_{\text {metric }}^{\mathrm{NR}} \cdot \Delta \phi$ remains remarkably small $(\sim \pm 0.02$ radians) during the entire inspiral and plunge ( $\omega_{2}=0.31$ being quite near the merger, see inset). By comparison, the root-sum of the various numerical errors on the phase (numerical truncation, outer boundary, extrapolation to infinity) is about 0.023 radians during the

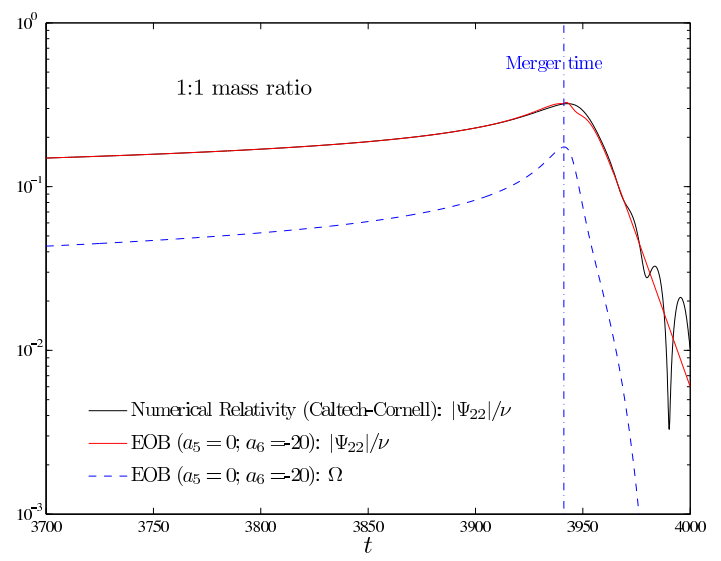

FIG. 3: Equal mass case: metric-amplitudes comparison. The maximum of the orbital frequency $\Omega$ defines the EOB merger.

inspiral [6]. At the merger, and during the ringdown, $\Delta \phi$ takes somewhat larger values ( $\sim \pm 0.1$ radians), but it oscillates around zero, so that, on average, it stays very well in phase with the NR waveform (as is clear on Fig. 11). By comparison, we note that [6] mentions that the phase error linked to the extrapolation to infinity doubles during ringdown. We also found that the NR signal after merger is contaminated by unphysical oscillations. We then note that the total "two-sigma" NR error level estimated in [6] rises to 0.05 radians during ringdown, which is comparable to the EOB-NR phase disagreement. Figure 3 compares the analytical and numerical metric moduli, $\left|\Psi_{22}\right| / \nu$. Again our (Padé-resummed, NQC-corrected) analytical waveform yields a remarkably accurate description of the inspiral NR waveform. During the early inspiral the fractional agreement between the moduli is at the $3 \times 10^{-3}$ level; as late as time $t=3900$, which corresponds to $1.5 \mathrm{GW}$ cycles before merger, the agreement is better than $1 \times 10^{-3}$. The discrepancy between the two moduli starts being visible only just before and just after merger (where it remains at the $2.5 \times 10^{-2}$ level). This very nice agreement should be compared with the previously considered EOB waveforms (which had a more primitive NQC factor, with $a_{2}=0[19,20]$ ) which led to large moduli disagreements $(\sim 20 \%$, see Fig. 9 in [20]) at merger. By contrast, the present moduli disagreement is comparable to the estimated NR modulus fractional error (whose two-sigma level is $2.2 \times 10^{-2}$ after merger [6]).

We also explored another aspect of the physical soundness of our analytical formalism: the triple comparison between (i) the NR GW energy flux at infinity (which was computed in 21]); (ii) the corresponding analytically predicted GW energy flux at infinity (computed by summing $\left|\dot{h}_{\ell m}\right|^{2}$ over $\ell, m$ ); and (iii) (minus) the mechanical energy loss of the system, as predicted by the general EOB formalism, i.e. the "work" done by the radiation reaction $\dot{E}_{\text {mechanical }}=\Omega \mathcal{F}_{\varphi}$. This comparison is shown in Fig. 4. which should be compared to Fig. 9 


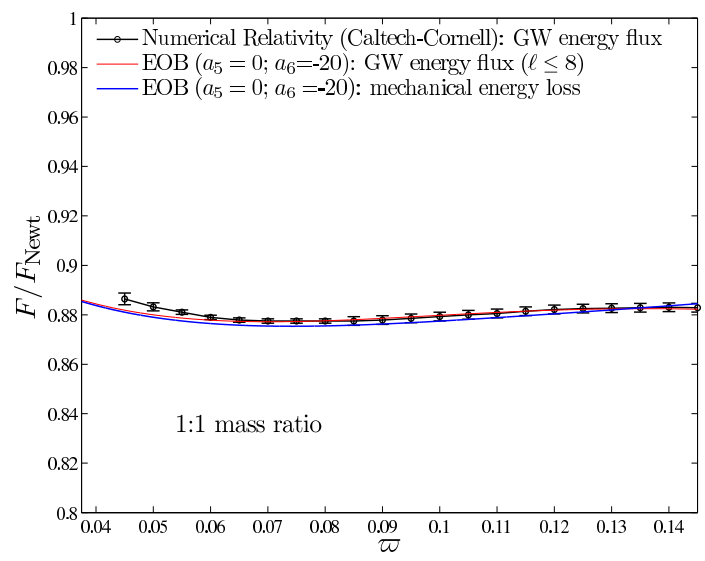

FIG. 4: Triple comparison between NR and EOB GW energy fluxes and the EOB mechanical energy loss.

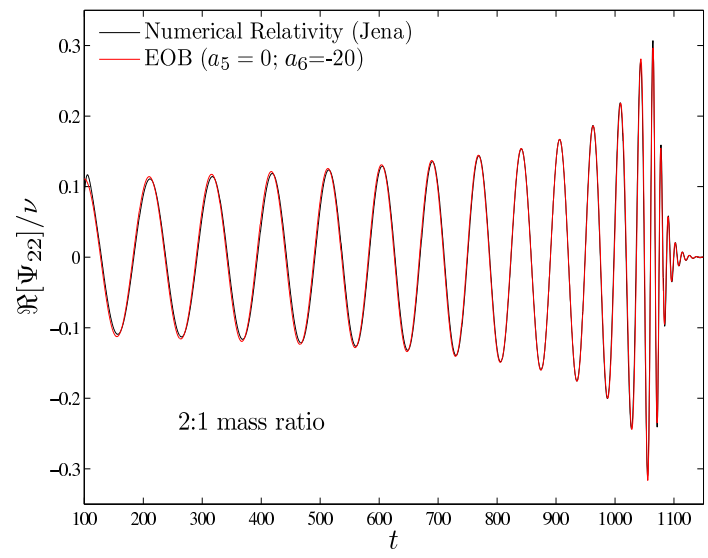

FIG. 5: Unequal mass case: Comparison between metric waveforms for the $2: 1$ mass ratio.

of 21]. We kept the same vertical scale as 21] which compared the NR flux to older versions of (resummed and non-resummed) analytical fluxes and needed such a $\pm 10 \%$ vertical scale to accomodate all the models they considered. [The horizontal axis is the frequency $\varpi$ of the differentiated metric waveform $\dot{h}_{22}$.] By contrast, we see again the striking closeness (at the $\sim 2 \times 10^{-3}$ level) between the analytical and NR GW fluxes. As both fluxes include higher multipoles than the $(2,2)$ one, this closeness is a further test of the agreement between our analytical formalism and NR results. [We think that the $\sim 2 \sigma$ difference between the (coinciding) analytical curves and the NR one on the left of the Figure is due to uncertainties in the flux computation of [21], possibly related to their method of computing $\dot{h}$.] Note that the rather close agreement between the analytical energy flux and the mechanical energy loss during late inspiral is not required by physics (because of the well-known "Schott term" [28]), but is rather an indication that $\dot{h}_{\ell m}$ can be well approximated by $-i m \Omega h_{\ell m}$ (used in Eq. (3)).

Finally, as the power of our formalism resides in the ease with which it can accomodate continuous variations in the basic physical parameters of the considered $\mathrm{BBH}$, we shall discuss an unequal-mass system $(\nu<1 / 4)$. The highest-accuracy data that we had in hands is the Jena-group simulation of a 2:1 mass ratio $\operatorname{BBH}(\nu=2 / 9=0.22222)$. When $a_{5}=0, a_{6}=-20$, and $\nu=2 / 9$, one finds $a_{1}=-0.017017, a_{2}=1.1906$. Using the data reported in 20] (and the function $\varphi(\nu)$ quoted above), we compare in Fig. 5 (the real part of) our analytical metric quadrupolar waveform $\Psi_{22}^{\mathrm{EOB}} / \nu$ to the corresponding (Jena, 2:1 mass ratio) NR metric waveform $\Psi_{22}^{\mathrm{NR}} / \nu$. [We use, as in [20], the pinching frequencies $\omega_{1}=0.1005, \omega_{2}=0.4542$.] Again we have an excellent analytical-numerical agreement, both in phase and in modulus. The small differences between the two are within the numerical errors (see [20]).

Conclusions. We have described a specific analytical formalism (within the EOB framework), which contains as arbitrariness only the resummation-flexibility parameters of the crucial EOB $A(u)$ potential. We have shown that for certain values [32] of these parameters $\left(a_{5}, a_{6}\right)$ : (i) the waveform predicted by our analytical formalism agrees, essentially within numerical errors, with the currently most accurate numerical relativity simulations; this agreement holds for several different mass ratios (1:1, 2:1 and 4:1 - not shown here); and (ii) the gravitational wave energy flux predicted by our formalism agrees, within numerical errors, with the most accurate numerical-relativity energy flux. We think that our formalism (possibly after some further minor improvements) opens a realistic possibility of constructing (with minimal computational resources) a very accurate, large bank of gravitational wave templates, thereby helping in both detecting and analyzing the signals emitted by inspiralling and coalescing binary black holes. [Though we have in mind essentially ground-based detectors, we think our method could also apply to space-based ones.] Finally, from a theoretical point of view, we think that our method can be extended to the description of (nearly circularized) spinning black hole systems (see [11]).

After the submission of this work, a paper [29] comparing Caltech-Cornell numerical data to a different version of the EOB formalism appeared on the archives. The EOB formalism of 29] does not use our novel (predictive) radiation reaction Eq. (3) (but rather the $v_{\text {pole }}{ }^{-}$ tuned one advocated in [18]), nor our $a_{6}$-improved $A$ potential, Eq. (21). Moreover, by contrast to the approach advocated here to reduce to an absolute minimum the number of adjusted parameters, namely two, $\left(a_{5}\right.$, $\left.a_{6}\right)$, Ref. 29] tunes six parameters: $\left(a_{5}(1 / 4), v_{\text {pole }}(1 / 4)\right.$, $a_{3}^{h_{22}}(1 / 4), a_{4}^{h_{22}}(1 / 4), a_{5}^{h_{22}}(1 / 4)$, and $\left.t_{\text {match }}^{22}(1 / 4)\right)$. 
[1] F. Pretorius, Phys. Rev. Lett. 95, 121101 (2005) arXiv:gr-qc/0507014.

[2] M. Campanelli, C. O. Lousto, P. Marronetti and Y. Zlochower, Phys. Rev. Lett. 96, 111101 (2006) arXiv:gr-qc/0511048.

[3] J. G. Baker, J. Centrella, D. I. Choi, M. Koppitz and J. van Meter, Phys. Rev. Lett. 96, 111102 (2006) arXiv:gr-qc/0511103.

[4] J. A. Gonzalez, U. Sperhake, B. Bruegmann, M. Hannam and S. Husa, Phys. Rev. Lett. 98, 091101 (2007) arXiv:gr-qc/0610154.

[5] M. Koppitz, D. Pollney, C. Reisswig, L. Rezzolla, J. Thornburg, P. Diener and E. Schnetter, Phys. Rev. Lett. 99, 041102 (2007) arXiv:gr-qc/0701163.

[6] M. A. Scheel, M. Boyle, T. Chu, L. E. Kidder, K. D. Matthews and H. P. Pfeiffer, Phys. Rev. D 79, 024003 (2009) arXiv:0810.1767 [gr-qc]]. Data available at http://www.black-holes.org/Waveforms.html

[7] F. Pretorius, arXiv:0710.1338 [gr-qc].

[8] A. Buonanno and T. Damour, Phys. Rev. D 59, 084006 (1999) arXiv:gr-qc/9811091.

[9] A. Buonanno and T. Damour, Phys. Rev. D 62, 064015 (2000) arXiv:gr-qc/0001013.

[10] T. Damour, P. Jaranowski and G. Schaefer, Phys. Rev. D 62, 084011 (2000) arXiv:gr-qc/0005034.

[11] T. Damour, Phys. Rev. D 64, 124013 (2001) arXiv:gr-qc/0103018.

[12] T. Damour, E. Gourgoulhon and P. Grandclement, Phys. Rev. D 66, 024007 (2002) arXiv:gr-qc/0204011.

[13] A. Buonanno, G. B. Cook and F. Pretorius, Phys. Rev. D 75, 124018 (2007) arXiv:gr-qc/0610122.

[14] T. Damour and A. Nagar, Phys. Rev. D 76, 044003 (2007) arXiv:0704.3550 [gr-qc]].

[15] Y. Pan et al., Phys. Rev. D 77, 024014 (2008) arXiv:0704.1964 [gr-qc]].

[16] T. Damour and A. Nagar, Phys. Rev. D 76, 064028 (2007) arXiv:0705.2519 [gr-qc]].

[17] A. Buonanno, Y. Pan, J. G. Baker, J. Centrella,
B. J. Kelly, S. T. McWilliams and J. R. van Meter, Phys. Rev. D 76, 104049 (2007) arXiv:0706.3732 [gr-qc]].

[18] T. Damour and A. Nagar, Phys. Rev. D 77, 024043 (2008) arXiv:0711.2628 [gr-qc]].

[19] T. Damour, A. Nagar, E. N. Dorband, D. Pollney and L. Rezzolla, Phys. Rev. D 77, 084017 (2008) arXiv:0712.3003 [gr-qc]].

[20] T. Damour, A. Nagar, M. Hannam, S. Husa and B. Bruegmann, Phys. Rev. D 78, 044039 (2008) arXiv:0803.3162 [gr-qc]].

[21] M. Boyle, A. Buonanno, L. E. Kidder, A. H. Mroue, Y. Pan, H. P. Pfeiffer and M. A. Scheel, arXiv:0804.4184 [gr-qc].

[22] M. Davis, R. Ruffini and J. Tiomno, Phys. Rev. D 5, 2932 (1972).

[23] T. Damour, P. Jaranowski and G. Schaefer, Phys. Lett. B 513, 147 (2001) arXiv:gr-qc/0105038.

[24] T. Damour, B. R. Iyer and A. Nagar, Phys. Rev. D 79, 064004 (2009) arXiv:0811.2069 [gr-qc].

[25] T. Damour, and A. Nagar, in preparation (2009).

[26] L. Blanchet, T. Damour, G. Esposito-Farese and B. R. Iyer, Phys. Rev. Lett. 93, 091101 (2004) arXiv:gr-qc/0406012.

[27] T. Tanaka, H. Tagoshi and M. Sasaki, Prog. Theor. Phys. 96, 1087 (1996) arXiv:gr-qc/9701050.

[28] G. A. Schott, Phil. Mag. 29, 49, (1915).

[29] A. Buonanno, Y. Pan, H. P. Pfeiffer, M. A. Scheel, L. T. Buchman and L. E. Kidder, arXiv:0902.0790 [grqc].

[30] Note that one could also similarly improve the subleading higher-multipolar-order contributions to $\mathcal{F}_{\varphi}$. We leave the exploration of such refinements to future work.

[31] Indeed, $a_{5}$ and $a_{6}$ formally parametrize the $4 \mathrm{PN}$ and $5 \mathrm{PN}$ contributions to the Taylor expansion of $A(u)$.

[32] We leave a detailed discussion of the "degenerate set" of "good" $\left(a_{5}, a_{6}\right)$ values to a future publication [25]. 